\title{
Alien hand syndrome without a corpus callosum lesion
}

\author{
Manfredi G Ventura, Serge Goldman, Jerzy Hildebrand
}

\begin{abstract}
A 58 year old right handed woman developed a left alien hand syndrome after a right capsulothalamic haemorrhage with mesencephalic extension. No lesion was detected in the corpus callosum. Positron emission tomography suggested that right cortical dysfunction might account for the absence of recognition of the left hand whereas involuntary motor activity was attributed to the lesion itself. This case shows that the alien hand syndrome is not always the expression of a disconnection syndrome and may occur after a large, strategically located, posterior lesion.
\end{abstract}

$(\mathcal{F}$ Neurol Neurosurg Psychiatry 1995;58:735-737)

Keywords: alien hand syndrome; corpus callosum; positron emission tomography

The alien hand syndrome was recently defined as a strange or foreign feeling of one's hand, failure to recognise its ownership without visual cue, together with observable involuntary motor activity. ${ }^{1}$ Originally Brion and Jedynak $^{2}$ described the alien hand syndrome in four patients with corpus callosum tumours. Since then all reported cases of alien hand syndrome have had, to our knowledge, some kind of corpus callosum lesion. ${ }^{1345}$ We describe a patient with the alien hand syndrome attributed to a unique lesion sparing the corpus callosum, and attempt to explain the symptomatology by anatomoclinical correlations with radiological findings and metabolic information provided by PET.

Université Libre de Bruxelles, Faculté de Médecine, CUB Hôpital Erasme, Brussels, Belgium Service de Neurologie M G Ventura J Hildebrand

Unité TEP/Cyclotron Biomédical $S$ Goldman

Correspondence to: Dr Manfredi G Ventura Service de Neurologie, CUB Hôpital Erasme, 808 route de Lennik, 1070 Brussels, Belgium.

Received 16 November 1994

Accepted 13 January 1995 deviation with upwards palsy, horizontal saccades, vergence paresis, and nystagmus retractorius. There was a left inferior facial palsy and left spastic hemiplegia, but full movements were possible on the right side. All tendon reflexes were elicited, the patellar and ankle reflexes being brisker on the left. Plantar responses were flexor on the right and extensor on the left. Pain was felt bilaterally but light touch was not perceived on the left side.

Consciousness returned gradually to normal within one week, and the patient became fully cooperative. Tonic eye deviation disappeared but upwards gaze palsy and saccadic horizontal eye movements persisted. Left hypotonia developed and strength improved to $4+/ 5$ in the left shoulder, to $2 / 5$ in the rest of the left upper limb, and to $3 / 5$ in the left lower limb. Ample, involuntary movements of the left upper limb, predominantly of the wrist and the hand, appeared. They occurred mainly as imitation synkineses during testing of right side strength and could not be reproduced voluntarily even with full concentration. The force of these movements was considerably superior compared with voluntary contractions. When recumbent, she also presented spontaneous left arm movements perceived as a levitation and described it as if "someone else was moving the arm". They occurred mainly at night and interfered with falling asleep. There was no grasp reflex on either side, no right side apraxia, no anosognosia, and no hemiasomatognosia.

Sensory examination was normal on the right side of her body but showed a moderate impairment of touch, pinprick, proprioceptive, and vibratory sensitivity on the left side. Dermolexia and stereognosia were abolished in the left hand. There was a left sided extinction to tactile double simultaneous stimulation. Moreover, the left hand placed in the right, either behind the patient's back or with eyes closed, was recognised as a hand but could not be differentiated from the examiner's or a third party's hand. The patient was able to cross localise tactile stimulation from the left to the right hand and vice versa.

Neuropsychological examination was normal except for visuoconstructive impairment shown on tests of spontaneous drawing or copying. There was no visual neglect.

Involuntary arm movements and nonrecognition of the left hand gradually vanished with day to day fluctuations, and 
Figure 1 (A) Sagittal T1 weighted image (TR 700 , TE $25 \mathrm{~ms}$, obtained $8 \mathrm{~mm}$ right from the midline) showing mesencephalic extension and the absence of lesion in the corpus callosum. (B) Axial T2 weighted MRI (TR 2700, TE $95 \mathrm{~ms}$ ) showing a high signal area in the right thalamus and in the posterior limb of the internal capsule.

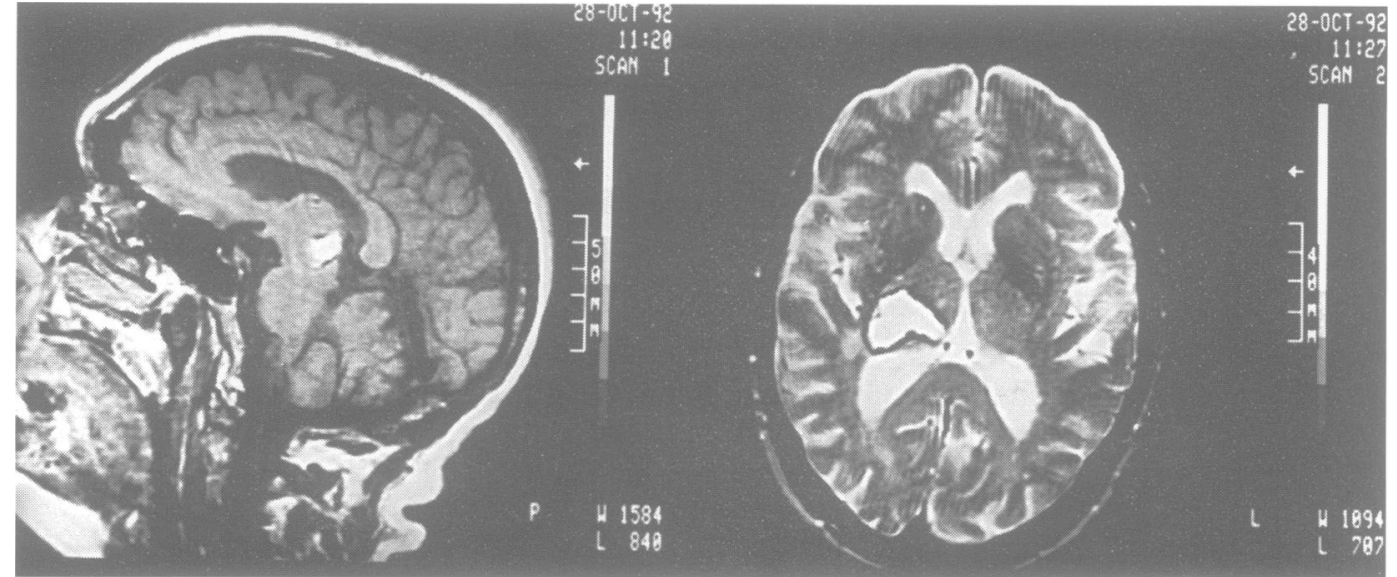

eventually resolved 10 weeks after onset. Three months after admission, at discharge, a discrete upgaze palsy was still present, strength was $4-15$ in the upper and $4+/ 5$ in the lower left limb, and the patient walked easily without support. Sensory extinction, stereognosia, and graphaesthesia abnormalities resolved completely but impairment of primary sensory modalities was still present.

Brain CT performed on admission showed a right capsulothalamic haemorrhage with mesencephalic extension, no mass effect toward the corpus callosum, and bilateral lenticular calcification. Controls of the CT performed two and three weeks later showed no modification. Magnetic resonance imaging was carried out four weeks after the haemorrhage, when the alien hand syndrome was still prominent. T1 and T2 weighted images showed a right capsulothalamic lesion involving the mesencephalon, the corpus callosum being unaffected (fig $1 \mathrm{~A}$ and $\mathrm{B}$ ).

Seven weeks after the cerebral haemorrhage, as the alien hand syndrome started to fluctuate, regional distribution of cerebral blood flow and cerebral glucose metabolism were studied by PET using $25 \mathrm{mCi}$ of ${ }^{15} \mathrm{O}$ $\mathrm{H}_{2} \mathrm{O}$ and $6 \mathrm{mCi}$ of ${ }^{18} \mathrm{~F}$-fluorodeoxyglucose (FDG) respectively. The procedure was carried out in a supine resting state with eyes
Figure 2 Upper images: PET with FDG images, on two successive planes through the fronto-parietal area, showing substantial asymmetry in cortical glucose metabolism particularly at the level of the sensory motor area. Lower images: PET with FDG images on two successive planes through the thalamus showing the severe thalamic glucose hypometabolism (except for the anterior part of the nucleus at the lower level) and the left temporal glucose hypometabolism. $R=$ right $L=$ left.

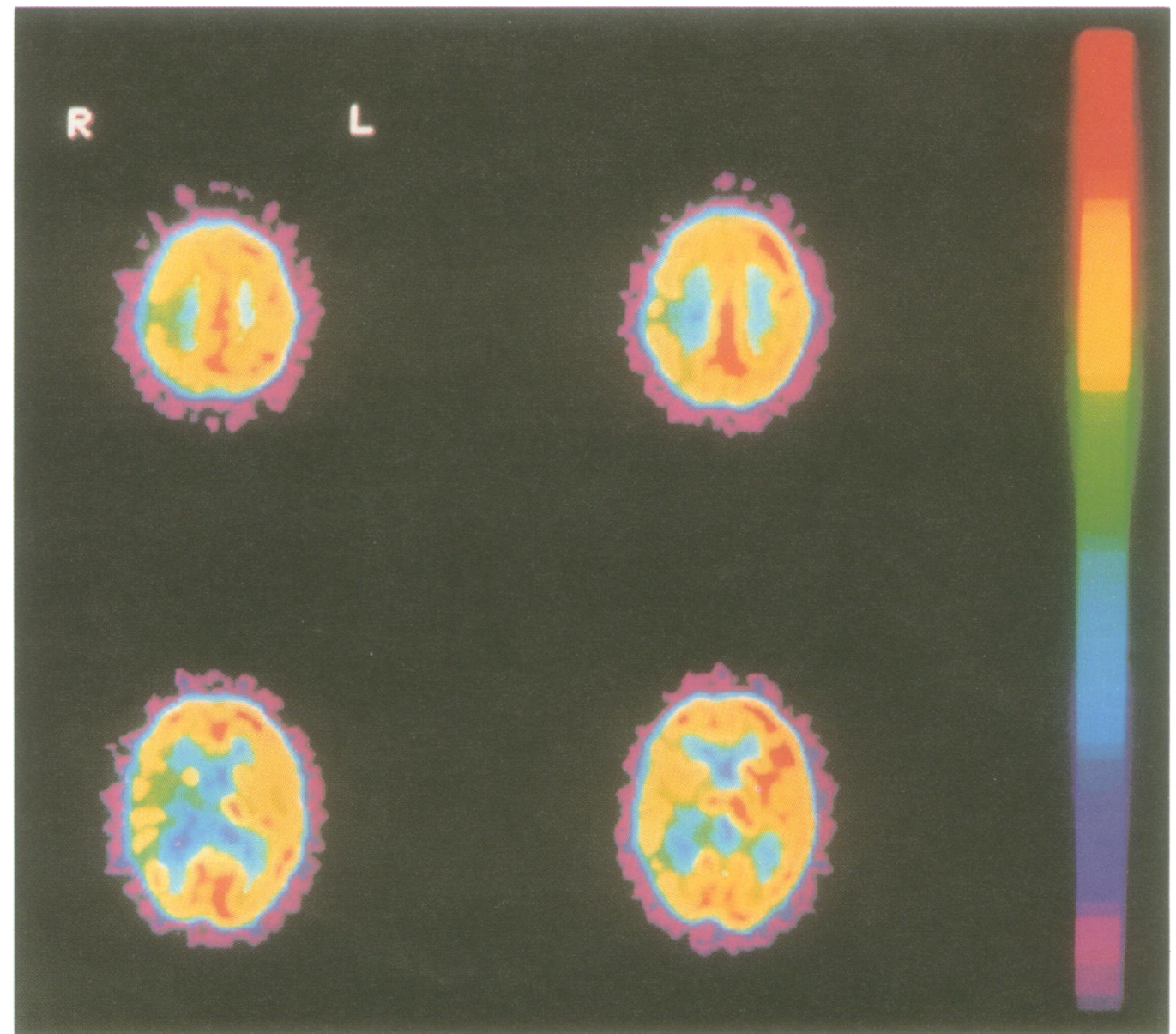


closed and ears unplugged. The two scans were performed on the same day. The PET tomograph was a CTI-Siemens 933-08-12 (Knoxville, TN), which provided $157 \mathrm{~mm}$ thick adjacent slices covering the entire brain. A transmission scan was obtained to perform the attenuation correction. Visual analysis of the ${ }^{15} \mathrm{O}-\mathrm{H}_{2} \mathrm{O}$ scan showed right thalamic hypoperfusion. The FDG scan showed low glucose metabolism in the posterior part of the right thalamus (fig 2). Glucose metabolism was also reduced in the right frontal, parietal, and temporal cortex, contrasting with an almost symmetric cortical blood flow at that level. Right cortical hypometabolism was the most pronounced in the sensorimotor area (fig 2).

\section{Discussion}

Our case fulfils the criteria of the alien hand syndrome given by Smith et al, ${ }^{1}$ by combining feeling of a foreign hand with involuntary movements. We attribute the alien hand syndrome in this patient to a right thalamic lesion. To the best of our knowledge, this is the first case of sustained alien hand syndrome reported without a lesion of the corpus callosum. ${ }^{235}$ Paroxysmal alien hand syndrome has recently been described in four patients without apparent damage to the corpus callosum and in these patients an ictal origin was suspected. ${ }^{6}$ Most cases of alien hand syndrome are generated by a combination of corpus callosum and frontal lesions. ${ }^{357}$ Experience gained from callosotomy shows that an isolated lesion of the corpus callosum produces only a temporary alien hand syndrome, which could partly result from transient injury caused by retraction of the medial frontal lobe during surgery. ${ }^{3}$ Posterior lesions with corpus callosum involvement have been reported in only two cases: patient 6 of Smith et $a l,{ }^{1}$ and the patient described by Levine and Rinn $^{4}$ who had an infarct of the right basal temporo-occipital lobe with minor ventral posterolateral thalamic and splenial involvement.

How can the right thalamic haemorrhage account for both the feeling of alienness and the involuntary movements presented by our patient? The absence of recognition of the left hand can be explained by the left tactile extinction, which resolved in parallel with the alien hand syndrome. Holding the left hand in the right produced bilateral simultaneous stimulation, thereby extinguishing tactile sensation originating from the left hand. The tac- tile loss on the left was only moderate and insufficient by itself to explain the foreignness of the hand.

Tactile extinction usually implies a parietal cortical lesion. In our case left parietal dysfunction, in the absence of CT or MRI abnormality, was shown by the presence of glucose hypometabolism at that level.

The thalamic and subthalamic location of the lesion could certainly account for the involuntary motor activity enhanced by contralateral voluntary movements. Indeed, both athetosis $^{8}$ and synkineses ${ }^{9}$ have been associated with lesions of this region.

Interestingly, the only neurological disorder where alien hand syndrome has been described without definite lesion of the corpus callosum is corticobasal ganglionic degeneration, ${ }^{10}$ a condition characterised by corticothalamic glucose hypometabolism ${ }^{11}$ similar to that in our patient.

In discussing the pathogenesis of alien hand syndrome, Smith et al stated that "a large posterior infarct alone may be sufficient to give an alien hand sign, although this has never been reported".' Our finding shows that this assumption proves to be correct and that the alien hand syndrome, usually considered as a manifestation of a disconnection syndrome, may result from a single strategically located lesion in the absence of involvement of the corpus callosum.

We thank Dr Martin L Albert, for his helpful comments in the preparation of this manuscript.

1 Smith Doody R, Jankovic J. The alien hand and related signs. I Neurol Neurosurg Psychiatry 1992;55:806-10.

2 Brion S, Jedynak CP. Troubles du transfert interhémisphérique. A propos de trois observations de tumeurs du corps calleux. Le signe de la main étrangère. Rev Neurol 1972;126:257-66.

3 Bogen JE. The callosal syndromes. In: Heilman KM, Valenstein E, eds. Clinical Neuropsychology. 2nd ed. New York: Oxford University Press, 1985:295-338.

4 Levine DN, Rinn WE. Opticosensory ataxia and alien hand syndrome after posterior cerebral artery territory infarction. Neurology 1986;36:1094-7.

5 Feinberg TE, Schindler RJ, Gilson Flanagan N, Haber LD. Two alien hand syndromes. Neurology 1992; 42:19-24.

6 Leiguarda $R$, Starkstein S, Nogués $M$, Berthier $M$, et al. Paroxysmal alien hand syndrome. $f$ Neurol Neurosurg Psychiatry 1993;56:788-92.

7 Tanaka Y, Iwasa H, Yoshida M. Diagnostic dyspraxia: case report and movement-related potentials. Neurology 1990;40:657-61

8 Milandre L, Brosset C, Gabriel B, Khalil R. Mouvements involontaires transitoires et infarctus thalamiques. Rev Neurol 1993;149:402-6.

9 Foix C, Hillemand P. Les syndrômes de la région thalamique. La Presse Médicale 1925;8:113-7.

10 Gibb WRG, Luthert PJ, Marsden CD. Corticobasal degeneration. Brain 1989;112:1171-92.

11 Eidelberg D, Dhawan V, Moeller JR, et al. The metabolic landscape of cortico-basal ganglionic degeneration: regional asymmetries studied with positron emission regional asymmetries studied with positron emission tomography 